\title{
Can pretreatment platelet-to-lymphocyte and neutrophil-to- lymphocyte ratios predict long-term oncologic outcomes after preoperative chemoradiation followed by surgery for locally advanced rectal cancer?
}

\author{
Sang Hyun An, Ik Yong Kim \\ Department of Surgery, Yonsei University Wonju College of Medicine, Wonju, Korea
}

Purpose: Systemic inflammation is associated with various malignancies, including colorectal cancer, as possible prognostic predictors. We aimed to evaluate the correlation of pretreatment the platelet-to-lymphocyte (PLR) and the neutrophil-tolymphocyte (NLR) ratio with long-term oncologic outcomes and pathologic complete response (pCR) in locally ad vanced rectal cancer patients who received neoadjuvant concurrent chemoradiotherapy (CRT) followed by curative resection. Methods: Between October 1996 and December 2015, 168 rectal cancer patients treated with preoperative CRT followed by surgery were enrolled. The set cut-off/mean PLR and NLR were 170 and 2.8. We analyzed the relationship between PLR, NLR, and the 5-year overall survival (OS), disease-free survival (DFS), and pCR rate.

Results: The 5-year OS rates were $75.9 \%$ and $59.8 \%$ in the highand low-PLR groups. The 5-year DFS rates were $62.9 \%$ and $50.8 \%$ in the high- and low-PLR groups, with no significant difference. In addition, the 5 -year OS rates were $75.7 \%$ and $58.4 \%$, and the 5-year DFS rates were $62.5 \%$ and $50.0 \%$ in the high- and low-NLR groups, respectively, both without any significant difference. Multivariate analysis showed only pretreatment PLR as an independent prognostic factor for OS (hazard ratio, 1.850; 95\% confidence interval, 1.041-3.287; $\mathrm{P}=0.036$ ), and both serologic markers were not independent prognostic factors for 5-year DFS.

Conclusion: Neither PLR nor NLR was associated with 5-year DFS nor pCR to neoadjuvant CRT. Only pretreatment PLR can be used in predicting OS in locally advanced rectal cancer patients who received neoadjuvant CRT followed by curative resection.

Keywords: Rectal neoplasms; Neoadjuvant therapy; Inflammation; Biomarkers; Survival

\section{INTRODUCTION}

Colorectal cancer (CRC) is the third most common malignancy and one of the leading causes of cancer-related deaths worldwide [1]. Specifically, rectal cancer accounts for $30 \%$ to $40 \%$ of CRCs,

Received: Jul 26, 2021 - Revised: Aug 29, 2021 - Accepted: Sep 6, 2021

Correspondence to: Ik Yong Kim, M.D., Ph.D.

Department of Surgery, Yonsei University Wonju College of Medicine, 20 Ilsan-ro, Wonju 26426, Korea

Tel: +82-33-741-0573, Fax: +82-33-741-0574

Email: iykim@yonsei.ac.kr

ORCID: https://orcid.org/0000-0001-6375-2216

(C) 2022 The Korean Society of Coloproctology

This is an open-access article distributed under the terms of the Creative Commons Attribution NonCommercial License (https://creativecommons.org/licenses/by-nc/4.0) which permits unrestricted noncommercial use, distribution, and reproduction in any medium, provided the original work is properly cited. and its treatment strategy is different and more complicated than that of colon cancer. Neoadjuvant chemoradiotherapy (CRT) followed by total mesorectal excision and adjuvant chemotherapy is the standard treatment strategy for locally advanced rectal cancer, which has led to a better prognosis of rectal cancer patients [2]. However, the 5-year overall survival (OS) of rectal cancer patients remains at approximately $60 \%$ [3]. To obtain more favorable oncologic outcomes, reliable markers predicting poor prognosis could help identify high-risk patients, allowing more careful follow-up and determining appropriate adjuvant treatment. The most well-known prognostic factor in rectal cancer patients is the TNM staging system proposed by the American Joint Committee on Cancer (AJCC). In addition, tumor markers, genetic information, and additional pathologic features, such as venous, lymphatic, and perineural invasion are used to predict tumor recur- 
rence and long-term prognosis. However, patients with similar clinicopathologic characteristics and staging may have different prognoses.

The inflammatory response of the host has become an interesting concern in recent clinical research. Systemic inflammation is known to play an important role in carcinogenesis and cancer progression, and several hematological markers can reflect the host's inflammatory status. In this context, there have been a number of studies investigating several pretreatment inflammatory laboratory markers have helped in diagnosing cancer and predicting short-term and long-term prognosis in the recent decade $[4,5]$. Such pretreatment laboratory markers can be easily assayed in routine blood tests, and their prognostic value for various types of cancer, including CRC, has been confirmed through previous studies $[4,6]$. Among various laboratory markers, the most frequently studied are neutrophils, lymphocytes, platelets, and their combinations. Platelet-to-lymphocyte (PLR) and neutrophil-to-lymphocyte (NLR) ratios have been widely used as prognostic tools in various malignancies, based on the theory that platelets and neutrophils are pro-tumorigenic factors and lymphocytes are protective factors against tumors $[7,8]$. Some studies have reported that elevated PLR and NLR can reflect poor oncologic outcomes as well as response to neoadjuvant CRT in rectal cancer patients $[9,10]$. However, there are still debates about the prognostic effect of PLR and NLR for locally advanced rectal cancer cases because of existing contradictory results $[9,11]$.

Therefore, we aimed to evaluate the long-term prognostic role of pretreatment PLR and NLR, as well as other hematological markers, for locally advanced rectal cancer patients who received neoadjuvant CRT followed by curative surgical resection. We also analyzed whether these serologic markers were correlated with pathologic tumor response to CRT. We hypothesized that elevated pretreatment PLR may be associated with poor long-term oncologic outcomes.

\section{METHODS}

\section{Patients}

We retrospectively reviewed data from 168 patients with histologically proven rectal adenocarcinoma who received neoadjuvant CRT followed by curative resection, between October 1996 and December 2015, at the Yonsei University Wonju Severance Christian Hospital. All patients underwent clinical evaluation, including digital rectal examination, basic laboratory tests, tumor marker assays, and colonoscopy. Computed tomography and pelvic magnetic resonance imaging (MRI) were used for clinical staging. The final diagnosis was confirmed by pathological reports. The exclusion criteria were as follows: metastatic rectal cancer at diagnosis (stage IV), emergency cases, palliative surgery, incomplete neoadjuvant chemotherapy or radiotherapy, and concomitant hematologic or coagulation disorders.

Informed consent was obtained from all patients and the study protocol was approved by the Institutional Review Board of Wonju Severance Christian Hospital (No. CR321067), in accordance with the Declaration of Helsinki.

\section{Hematologic markers}

Peripheral blood samples were obtained 1 week prior to neoadjuvant CRT and 1 week prior to the index surgery. White blood cell (WBC), neutrophil, lymphocyte, and platelet counts, along with albumin and C-reactive protein levels, were included. The PLR and NLR were determined by dividing the absolute platelet and neutrophil count, respectively, by the absolute lymphocyte count. The cut-off PLR and NLR were also set as the mean values.

\section{Outcomes}

The primary outcomes were 5-year OS and disease-free survival (DFS). The secondary outcome was the pathologic response to neoadjuvant CRT. OS was defined as the date of diagnosis to the date of all-cause death, and DFS was defined as the date of diagnosis to the date of recurrence or all-cause death. Pathologic complete response (pCR) was defined as the absence of residual invasive cancer cells in resected specimens and harvested lymph nodes.

\section{Treatment protocol and follow-up}

All included patients received a total radiation dose of $50.4 \mathrm{~Gy}$ over 5 weeks ( $45 \mathrm{~Gy}$ in 25 fractions to the whole pelvis and $5.4 \mathrm{~Gy}$ in 3 fractions to the primary tumor). Concurrent chemotherapy was administered via an intravenous bolus of 5-fluorouracil (425 $\mathrm{mg} / \mathrm{m}^{2} /$ day) and leucovorin $\left(20 \mathrm{mg} / \mathrm{m}^{2} /\right.$ day $)$ for 5 days during the 1 st and 5th weeks of radiation therapy, or via oral capecitabine $\left(1,650 \mathrm{mg} / \mathrm{m}^{2} /\right.$ day $)$, twice daily, during the whole radiation therapy period. Post-CRT pelvic MRI was performed for restaging. Six to 8 weeks after the completion of neoadjuvant CRT (mean duration, 7.6 weeks), curative total mesorectal excision was performed by 2 experienced colorectal surgeons. The pathologic (yp) stage was recorded according to the eighth edition of the AJCC TNM classification. Postoperative adjuvant chemotherapy was administered using intravenous 5 -fluorouracil $\left(425 \mathrm{mg} / \mathrm{m}^{2}\right)$ and leucovorin $\left(20 \mathrm{mg} / \mathrm{m}^{2}\right)$ for 4 to 8 weeks. Patients were followed up every 3 months for the first 2 years postoperatively, semiannually for the next 3 years, and annually thereafter. Physical examinations and laboratory tests for tumor markers were performed at each follow-up, and abdominopelvic computed tomography was performed semiannually. Colonoscopy was performed annually or when the patient had recurrent or other abnormal symptoms. Patients were followed up for 5 years and additional diagnostic tests, such as MRI, were performed for suspected recurrence or metastasis.

\section{Statistical analysis}

Categorical variables were described by frequencies and percentages and were analyzed using the chi-square test or Fisher exact 
test. Continuous variables were described as means and standard deviations and were analyzed using the Student t-test or MannWhitney U-test. Kaplan-Meier curve analysis with log-rank test and the Cox proportional hazard model were used for survival analysis. Variables in multivariate analysis were selected by backward stepwise method. All statistical analyses were performed us-

Table 1. Baseline characteristics of the patients

\begin{tabular}{|c|c|}
\hline Characteristic & Data \\
\hline Age (yr) & $61.06 \pm 10.76$ \\
\hline$<65$ & $97(59.9)$ \\
\hline$\geq 65$ & $65(40.1)$ \\
\hline \multicolumn{2}{|l|}{ Sex } \\
\hline Male & $121(74.7)$ \\
\hline Female & $41(25.3)$ \\
\hline Tumor location from anal verge (cm) & $6.34 \pm 3.26$ \\
\hline \multicolumn{2}{|l|}{ Carcinoembryonic antigen (ng/mL) } \\
\hline Pre-CRT & $8.41 \pm 16.93$ \\
\hline Post-CRT & $4.05 \pm 6.74$ \\
\hline \multicolumn{2}{|l|}{ Operation } \\
\hline LAR & $127(78.4)$ \\
\hline Hartmann & $8(4.9)$ \\
\hline APR & $25(15.4)$ \\
\hline Local excision & $1(0.6)$ \\
\hline \multicolumn{2}{|l|}{ Diverting stoma } \\
\hline None & $14(8.6)$ \\
\hline lleostomy & $116(71.6)$ \\
\hline Colostomy & $32(19.8)$ \\
\hline Tumor size $(\mathrm{cm})$ & $3.112 \pm 1.71$ \\
\hline No. of harvested lymph nodes & $16.61 \pm 8.68$ \\
\hline \multicolumn{2}{|l|}{ Stage } \\
\hline 0 & $17(10.5)$ \\
\hline I & $39(24.1)$ \\
\hline$\|$ & $58(35.8)$ \\
\hline III & $48(29.6)$ \\
\hline \multicolumn{2}{|l|}{ Tumor differentiation } \\
\hline WD & $30(18.5)$ \\
\hline MD & $120(74.1)$ \\
\hline PD, mucinous & $8(4.9)$ \\
\hline \multicolumn{2}{|l|}{ Pathologic complete response } \\
\hline Yes & $17(10.5)$ \\
\hline No & $144(88.9)$ \\
\hline
\end{tabular}

Values are presented as mean \pm standard deviation or number (\%).

CRT, chemoradiotherapy; LAR, low anterior resection; APR, abdominoperineal resection; WD, well differentiation; MD, moderate differentiation; PD, poorly differentiation. ing IBM SPSS Statistics for Windows ver. 25.0 (IBM Corp., Armonk, NY, USA), and the P-values of $<0.05$ were considered statistically significant.

\section{RESULTS}

\section{Patient characteristics}

Among 168 patients with locally advanced rectal cancer who underwent neoadjuvant CRT, 2 with hematologic disorders and 4 who underwent palliative surgery were excluded. Among the 162 included patients, the median follow-up period was 66 months (range, 0-234 months), the median age was 61 years (range, 3885 years), and 121 (74.7\%) were male. The pre- and post-CRT mean serum carcinoembryonic antigen (CEA) levels were 8.41 $\mathrm{ng} / \mathrm{mL}$ and $4.05 \mathrm{ng} / \mathrm{mL}$, respectively. Low anterior resection was the most commonly performed operation (78.9\%), and approximately $71.6 \%$ of the patients underwent protective diversion to ensure a safe anastomosis site. The tumors were located at a mean of $6.34 \mathrm{~cm}$ above the anal verge, with a mean size of $3.11 \mathrm{~cm}$. After preoperative CRT, 17 patients $(10.5 \%)$ showed pCR. The stages after surgery were as follows: stage I, 9.9\% $(n=17)$; stage II, $24.1 \%(\mathrm{n}=39)$; and stage III, $29.6 \%(\mathrm{n}=48)$ (Table 1$)$.

\section{Laboratory profiles}

The mean WBC, neutrophil, lymphocyte, and platelet counts were $7.4 \times 10^{9} / \mathrm{L}, 4.7 \times 10^{9} / \mathrm{L}, 1.9 \times 10^{9} / \mathrm{L}$, and $300 \times 10^{9} / \mathrm{L}$, respectively. The mean serum albumin and C-reactive protein levels were $4.13 \mathrm{~g} / \mathrm{dL}$ and $1.06 \mathrm{mg} / \mathrm{dL}$, respectively. The mean/cut-off NLR and PLR used were 2.80 and 170, respectively (Table 2).

\section{Relationships between clinicopathologic serologic factors and pathologic complete response to neoadjuvant chemoradiotherapy}

On univariate analysis, pCR to neoadjuvant CRT was not associated with clinicopathologic features such as age, sex, tumor differentiation, or tumor location. Tumor size was significantly smaller in the group showing pCR $(1.72 \pm 0.78 \mathrm{~cm}$ vs. $3.22 \pm 1.72 \mathrm{~cm}$,

Table 2. Pretreatment laboratory markers

\begin{tabular}{lcc}
\hline Laboratory profile & Mean \pm 2 SD & Median (IQR) \\
\hline WBC & $7,426.63 \pm 2,086.62$ & $7,510(6,020-8,845)$ \\
Neutrophil & $4,709.00 \pm 1,720.24$ & $4,500(3,520-5,520)$ \\
Lymphocyte & $1,986.63 \pm 732.25$ & $1,970(1,510-2,360)$ \\
Platelet & $300,088.05 \pm 86,951.05$ & $294,500(239,750-350,500)$ \\
Albumin & $4.13 \pm 0.49$ & $4.2(3.9-4.5)$ \\
C-reactive protein & $1.06 \pm 2.41$ & $0.29(0.14-0.615)$ \\
NLR & $2.80 \pm 2.92$ & $2.31(1.69-3.21)$ \\
PLR & $170.28 \pm 98.65$ & $152(114-202)$ \\
\hline
\end{tabular}

SD, standard deviation; IQR, interquartile range; WBC, white blood cell; NLR, neutrophil-to-lymphocyte ratio; PLR, platelet-to-lymphocyte ratio. 
$\mathrm{P}=0.005)$. There was no association between $\mathrm{pCR}$ and serologic markers, including WBC, neutrophil, lymphocyte, and platelet counts, and serum albumin and C-reactive protein levels. In addition, $\mathrm{pCR}$ to neoadjuvant $\mathrm{CRT}$ did not show any association with pre-CRT PLR and NLR $(\mathrm{P}=0.799$ and $\mathrm{P}=0.422$, respectively), but was significantly associated with the pre-CRT and post-CRT CEA levels $(4.16 \pm 3.92 \mathrm{ng} / \mathrm{mL}$ vs. $8.90 \pm 17.86 \mathrm{ng} / \mathrm{mL}, \mathrm{P}=0.008$; and $2.23 \pm 0.35 \mathrm{ng} / \mathrm{mL}$ vs. $4.86 \pm 7.97 \mathrm{ng} / \mathrm{mL}, \mathrm{P}=0.001$; respectively) (Table 3).

\section{Survival analysis}

During the 5-year follow-up period, there were 15 cases of local recurrence (9.3\%) and 37 cases of systemic recurrence (22.8\%). In addition, the overall 5-year OS and DFS rates were $70.4 \%$ and $58.6 \%$, respectively. According to the Kaplan-Meier survival analysis, the 5-year OS rates were significantly different between the high- and low-PLR groups (75.9\% vs. 59.8\%, $\mathrm{P}=0.033$ ). However, the 5-year DFS rates were not significantly different between the 2 groups ( $62.9 \%$ vs. $50.8 \%, P=0.125$ ). In terms of NLR, the 5 -year OS rates were $75.7 \%$ in high NLR group and $58.4 \%$ in low NLR group $(\mathrm{P}=0.035)$; and 5 -year DFS rates were $62.0 \%$ in high NLR group and $50.0 \%$ in low NLR group, respectively $(\mathrm{P}=0.173$, respectively) (Fig. 1). In addition, we further analyzed the correlation between survival and inflammatory markers by dividing pa-

Table 3. The association between pathologic complete response (pCR) and serologic markers

\begin{tabular}{|c|c|c|c|}
\hline Variable & $\mathrm{pCR}(\mathrm{n}=17)$ & Non-pCR $(n=145)$ & P-value \\
\hline \multicolumn{4}{|l|}{ Age (yr) } \\
\hline$<65$ & $10(58.8)$ & $86(59.7)$ & \\
\hline$\geq 65$ & $7(41.2)$ & $58(40.3)$ & $>0.999$ \\
\hline \multicolumn{4}{|l|}{ Sex } \\
\hline Male & $10(58.8)$ & $110(76.4)$ & \\
\hline Female & $7(41.2)$ & $34(23.6)$ & $0.142^{\mathrm{a}}$ \\
\hline \multicolumn{4}{|l|}{ Tumor differentiation } \\
\hline WD & $2(11.8)$ & $28(19.9)$ & \\
\hline MD & 15 (88.2) & $105(74.5)$ & \\
\hline PD, Mucinous & $0(0)$ & $8(5.7)$ & $0.541^{a}$ \\
\hline Tumor location from anal verge (cm) & $5.94 \pm 3.27$ & $6.39 \pm 3.27$ & 0.604 \\
\hline Tumor size (cm) & $1.72 \pm 0.78$ & $3.22 \pm 1.72$ & $0.005^{b}$ \\
\hline White blood cell $\left(\times 10^{9} / L\right)$ & $7,498.24 \pm 1,708.019$ & $7,392.25 \pm 2,117.024$ & 0.843 \\
\hline Neutrophil $\left(\times 10^{9} / \mathrm{L}\right)$ & $4,713.53 \pm 1,666.437$ & $4,684.30 \pm 1,714.003$ & 0.947 \\
\hline Lymphocyte (×109/L) & $1,982.35 \pm 362.793$ & $1,985.99 \pm 767.797$ & 0.974 \\
\hline C-reactive protein (mg/dL) & $0.48 \pm 0.50$ & $1.08 \pm 2.52$ & 0.430 \\
\hline Albumin (g/dL) & $4.282 \pm 0.354$ & $4.118 \pm 0.506$ & 0.195 \\
\hline \multicolumn{4}{|l|}{ Platelet (×109/L) } \\
\hline$<300$ & $8(47.1)$ & $77(54.6)$ & \\
\hline$\geq 300$ & $9(52.9)$ & $64(45.4)$ & 0.613 \\
\hline \multicolumn{4}{|l|}{ PLR } \\
\hline$<170$ & $11(64.7)$ & $86(60.6)$ & \\
\hline$\geq 170$ & $6(35.3)$ & $56(39.4)$ & 0.799 \\
\hline \multicolumn{4}{|l|}{ NLR } \\
\hline$<2.8$ & $13(76.5)$ & $91(64.1)$ & \\
\hline$\geq 2.8$ & $4(23.5)$ & $51(35.9)$ & 0.422 \\
\hline Pre-CRT CEA (ng/mL) & $4.157 \pm 3.916$ & $8.903 \pm 17.860$ & 0.008 \\
\hline Post-CRT CEA (ng/mL) & $2.23 \pm 0.349$ & $4.86 \pm 7.93$ & 0.001 \\
\hline
\end{tabular}

WD, well differentiation; MD, moderate differentiation; PD, poorly differentiation; PLR, plate-to-lymphocyte ratio; NLR, neutrophil-to-lymphocyte ratio; CRT, chemoradiotherapy; CEA, carcinoembryonic antigen.

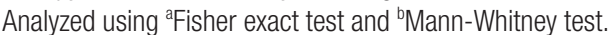


tients into 3 groups, as follows: group A, with lower PLR and NLR than the cut-off values; group $\mathrm{B}$, with only one marker lower than the cut-off value, and group C: with higher PLR and NLR than the cut-off values. The 5-year OS rates were 77.2\%, 69.0\%, and $55.7 \%$ in groups A, B, and C, respectively, with statistically significant differences between groups $\mathrm{A}$ and $\mathrm{C}$, but not group $\mathrm{B}(\mathrm{P}=$
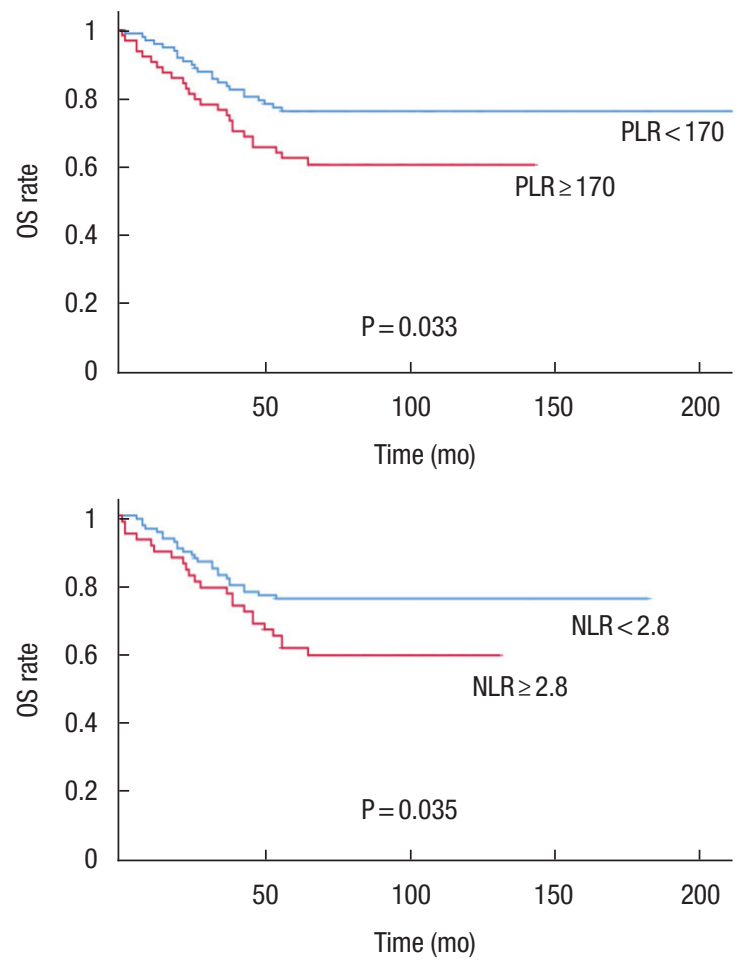

0.019). The 5-year DFS rates were 66.3\%, 59.0\%, and 53.7\% in groups $\mathrm{A}, \mathrm{B}$, and $\mathrm{C}$, respectively, with no statistically significant intergroup differences $(\mathrm{P}=0.250)$ (Fig. 2).

Univariate and multivariate analyses using the Cox proportional hazard model were performed to identify independent prognostic factors for OS and DFS. Univariate analysis showed that stage III
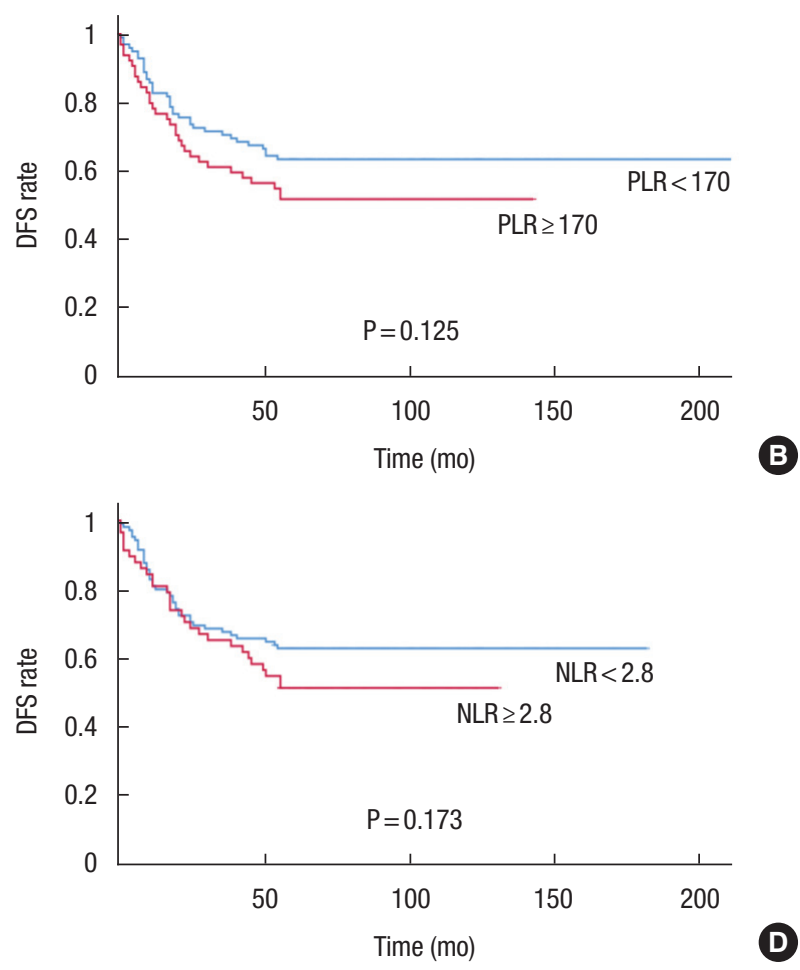

Fig. 1. Survival rates according to laboratory markers. (A) The 5-year overall survival (OS) rates were $75.9 \%$ and $59.8 \%$ in the high- and lowPLR groups, respectively $(\mathrm{P}=0.033)$. (B) The 5 -year disease-free survival (DFS) rates were $62.9 \%$ and $50.8 \%$ in the high- and low-PLR groups, respectively $(\mathrm{P}=0.125)$. (C) The 5 -year OS rates were $75.7 \%$ and $58.4 \%$ in the high- and low-NLR groups, respectively $(\mathrm{P}=0.035)$. (D) The 5 -year DFS rates were $62.5 \%$ and $50.0 \%$ in the high- and low-NLR groups, respectively $(\mathrm{P}=0.173)$. PLR, platelet-to-lymphocyte ratio; NLR, neutrophil-to-lymphocyte ratio.
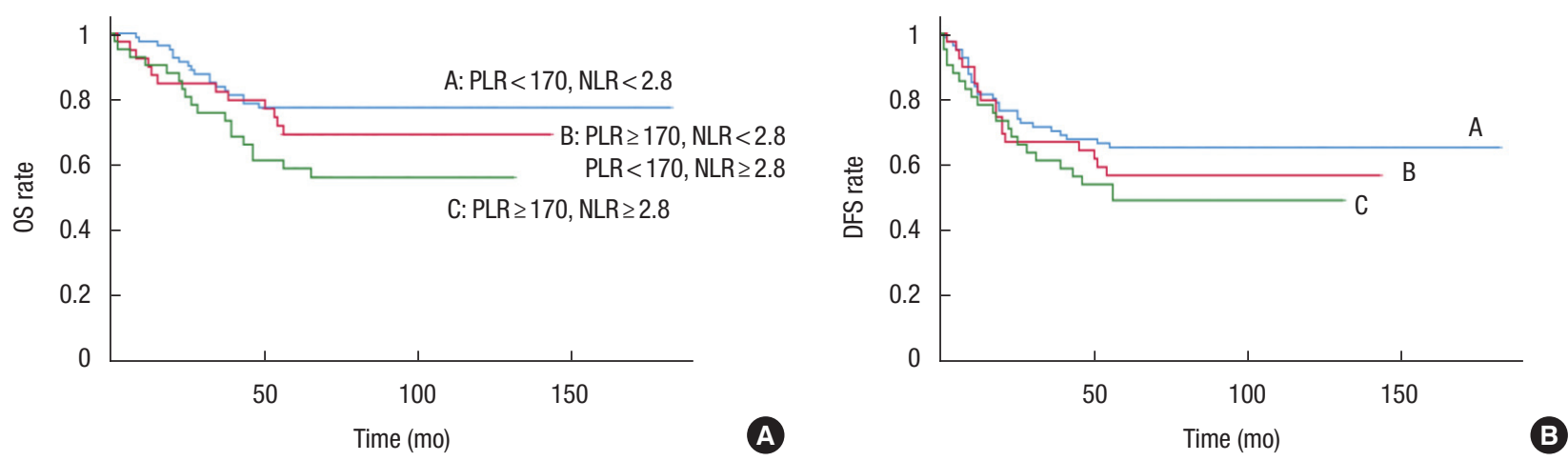

Fig. 2. Survival curves according to the number of elevated laboratory markers. (A) The 5-year overall survival (OS) rates were $77.2 \%, 69.0 \%$, and $55.7 \%$ in groups $\mathrm{A}, \mathrm{B}$, and $\mathrm{C}$, respectively, with statistically significant differences between groups $\mathrm{A}$ and $\mathrm{C}$, but not group $\mathrm{B}(\mathrm{P}=0.019$ ). (B) The 5-year disease-free survival (DFS) rates were $66.3 \%, 59 \%$, and $53.7 \%$ in groups A, B, and C, respectively $(\mathrm{P}=0.250)$. PLR, platelet-tolymphocyte ratio; NLR, neutrophil-to-lymphocyte ratio. 
Table 4. Cox regression analysis for 5-year overall survival

\begin{tabular}{|c|c|c|c|c|c|}
\hline \multirow{2}{*}{ Variable } & \multirow{2}{*}{ Category } & \multicolumn{2}{|c|}{ Univariate analysis } & \multicolumn{2}{|c|}{ Multivariate analysis } \\
\hline & & $\mathrm{HR}(95 \% \mathrm{Cl})$ & P-value & $\mathrm{HR}(95 \% \mathrm{Cl})$ & P-value \\
\hline Age (yr) & $<65$ vs. $\geq 65$ & $1.001(0.561-1.786)$ & 0.997 & & \\
\hline Sex & Male vs. female & $1.163(0.615-2.198)$ & 0.643 & & \\
\hline Stage & 0, I, II vs. III & $2.535(1.428-4.499)$ & 0.001 & $2.445(1.360-4.396)$ & 0.003 \\
\hline $\mathrm{pCR}$ & Yes vs. No & 1.099 (0.626-1.928) & 0.743 & & \\
\hline Albumin (g/dL) & $<3.5$ vs. $\geq 3.5$ & $1.486(0.631-3.501)$ & 0.365 & & \\
\hline Platelet $\left(\times 10^{9} / L\right)$ & $<300$ vs. $\geq 300$ & $1.071(0.608-1.887)$ & 0.812 & & \\
\hline PLR & $<170$ vs. $\geq 170$ & $1.833(1.040-3.230)$ & 0.036 & $1.786(1.007-3.169)$ & 0.047 \\
\hline NLR & $<2.8$ vs. $\geq 2.8$ & 1.819 (1.032-3.205) & 0.038 & & \\
\hline Pre-CRT CEA (ng/mL) & $<5$ vs. $\geq 5$ & $1.556(0.881-2.746)$ & 0.128 & & \\
\hline Post-CRT CEA (ng/mL) & $<5$ vs. $\geq 5$ & $3.492(1.866-6.533)$ & $<0.001$ & $2.780(1.440-5.365)$ & 0.002 \\
\hline
\end{tabular}

$\mathrm{HR}$, hazard ratio; $\mathrm{Cl}$, confidence interval; $\mathrm{pCR}$, pathologic complete response; PLR, plate-to-lymphocyte ratio; NLR, neutrophil-to-lymphocyte ratio; CRT, chemoradiotherapy; CEA, carcinoembryonic antigen.

Table 5. Cox regression analysis for 5-year disease-free survival

\begin{tabular}{|c|c|c|c|c|c|}
\hline \multirow{2}{*}{ Variable } & \multirow{2}{*}{ Category } & \multicolumn{2}{|c|}{ Univariate } & \multicolumn{2}{|c|}{ Multivariate } \\
\hline & & $\mathrm{HR}(95 \% \mathrm{Cl})$ & P-value & $\mathrm{HR}(95 \% \mathrm{Cl})$ & P-value \\
\hline Age (yr) & $<65$ vs. $\geq 65$ & $1.086(0.669-1.765)$ & 0.738 & & \\
\hline Sex & Male vs. female & $1.043(0.601-1.808)$ & 0.881 & & \\
\hline Stage & 0, I, II vs. III & $2.225(1.360-3.640)$ & 0.001 & $2.177(1.319-3.595)$ & 0.002 \\
\hline $\mathrm{pCR}$ & Yes vs. No & $1.114(0.619-2.008)$ & 0.718 & & \\
\hline Albumin (g/dL) & $<3.5$ vs. $\geq 3.5$ & $0.889(0.284-2.057)$ & 0.783 & & \\
\hline Platelet $\left(\times 10^{9} / L\right)$ & $<300$ vs. $\geq 300$ & $1.320(0.818-2.132)$ & 0.256 & & \\
\hline PLR & $<170$ vs. $\geq 170$ & $1.451(0.898-2.346)$ & 0.129 & & \\
\hline NLR & $<2.8$ vs. $\geq 2.8$ & $1.396(0.859-2.269)$ & 0.178 & & \\
\hline Pre-CRT CEA (ng/mL) & $<5$ vs. $\geq 5$ & 1.749 (1.082-2.826) & 0.022 & & \\
\hline Post-CRT CEA (ng/mL) & $<5$ vs. $\geq 5$ & $3.500(2.006-6.109)$ & $<0.001$ & $3.291(1.853-5.845)$ & $<0.001$ \\
\hline
\end{tabular}

HR, hazard ratio; $\mathrm{Cl}$, confidence interval; $\mathrm{pCR}$, pathologic complete response; PLR, plate-to-lymphocyte ratio; NLR, neutrophil-to-lymphocyte ratio; CRT, chemoradiotherapy; CEA, carcinoembryonic antigen.

(hazard ratio [HR], 2.535; 95\% confidence interval [CI], 1.2484.499; $\mathrm{P}=0.001$ ), PLR $\geq 170$ (HR, 1.833; 95\% CI, 1.040-3.230; $\mathrm{P}=0.036), \mathrm{NLR} \geq 2.8$ (HR, 1.819; 95\% CI, 1.032-3.205; $\mathrm{P}=0.038$ ), and post-CRT CEA level $\geq 5 \mathrm{ng} / \mathrm{mL}$ (HR, 3.492; 95\% CI, 1.866-6.533; $\mathrm{P}<0.001)$ were statistically significant prognostic factors for worse OS. On multivariate analysis, stage III (HR, 2.445; 95\% CI, 1.360-4.396, $\mathrm{P}=0.003), \mathrm{PLR} \geq 170$ (HR, 1.786; 95\% CI, 1.007-3.169; $\mathrm{P}=0.047)$, and post-CRT CEA level $\geq 5 \mathrm{ng} /$ $\mathrm{mL}$ (HR, 2.780; 95\% CI 1.440-5.365; $\mathrm{P}=0.002)$ were independent poor prognostic factors for OS (Table 4 ). In addition, univariate analysis for DFS showed that stage III (HR, 2.225; 95\% CI, 1.3603.640; $\mathrm{P}=0.001$ ), pre-CRT CEA level $\geq 5 \mathrm{ng} / \mathrm{mL}$ (HR, 1.749; 95\% CI, 1.082-2.826; $\mathrm{P}=0.022$ ), and post-CRT CEA level $\geq 5 \mathrm{ng} / \mathrm{mL}$ (HR, 3.500; 95\% CI, 2.006-6.109; $\mathrm{P}<0.001$ ) were significant poor prognostic factors. Multivariate analysis showed that stage III
(HR, 2.177; 95\% CI, 1.319-3.595; $\mathrm{P}=0.002$ ) and post-CRT CEA level $\geq 5 \mathrm{ng} / \mathrm{mL}$ (HR, 3.291; 95\% CI, 1.853-5.845; P < 0.001) were independent prognostic factors for poor DFS (Table 5 ).

\section{DISCUSSION}

We investigated the prognostic role of pretreatment PLR and NLR in patients with locally advanced rectal cancer who received neoadjuvant CRT followed by curative resection. In summary, a smaller tumor size and lower CEA level, but not pretreatment PLR and NLR, were associated with pCR to neoadjuvant CRT. In terms of oncologic outcomes, pretreatment high PLR and NLR were associated with poor 5-year OS on univariate analysis, but only pretreatment high PLR was an independent prognostic factor on multivariate analysis. However, neither serologic inflam- 
matory marker was associated with 5-year DFS.

The mechanisms underlying the relationship between systemic inflammation and tumor biology are not yet fully understood; to our knowledge, chronic inflammation induces tissue damage, and repeated regeneration processes result in permanent genetic mutations, such as point mutations, deletions, or rearrangements. Activated inflammatory cells produce numerous chemokines and cytokines, which can influence tumor growth, migration, and differentiation by releasing growth factors. Platelets, which stop bleeding by adhering and aggregating at the injured tissue area, also play important roles in host inflammation and the immune system $[12,13]$. Increased and activated platelets release growth factors that facilitate tumor growth and invasion, and contribute to tumor metastasis by helping in cancer cell adhesion and extravasation [14]. Thrombocytosis is associated with the long-term prognosis of CRC patients $[15,16]$. Lymphocytes are a subtype of WBCs that are responsible for innate immunity. Lymphocytes are known to play a key role in fighting tumor progression, and a high density of lymphocytic infiltration of the tumor is a known prognostic factor for improved survival in numerous malignancies [17]. Neutrophils are the most abundant WBCs and play an important role in the acute inflammatory response. In addition, neutrophils are associated with various carcinogenic processes, such as tumor growth and proliferation, and tumor angiogenesis by releasing reactive oxygen and nitrogen species, or proteases. Neutrophils can also cause metastatic spread by suppressing natural killer functions and promoting extravasation of tumor cells [18].

In our analysis, 17 patients $(10.5 \%)$ showed a pCR to neoadjuvant CRT, which is consistent with previously published data. pCR after neoadjuvant CRT is associated with favorable longterm outcomes $[19,20]$. Patients with pCR showed a lower incidence of local recurrence and distant metastasis, and higher longterm survival. Sell et al. [19] analyzed the results of 10-year followup for stage II to III rectal cancer patients, and reported that patients who achieved pCR after neoadjuvant CRT showed excellent 10-year DFS and OS. In this context, there are many studies on organ-preserving approaches, such as wait-and-watch, or local excision suggesting that a pCR has been acquired after neoadjuvant CRT through imaging and endoscopic studies $[21,22]$. According to previous literature, organ-preserving approaches have been determined as feasible and comparable in terms of longterm survival, even if salvage surgery was performed because of recurrence during the close follow-up period. However, in our analysis, the association between pCR and survival was not demonstrated, probably because the number of cases was small.

In addition to imaging tests, an organ-preserving treatment strategy may determine robust prognostic predictors that can help evaluate the response to neoadjuvant CRT. In our analysis, the correlation between the pretreatment serologic markers and pCR was not confirmed, and only tumor size and CEA level were confirmed to be related to $\mathrm{pCR}$. There have been several studies on whether inflammatory serologic markers are useful in predicting pCR, but there is still debate [10, 23]. Policicchio et al. [24] reported that a high platelet count $\left(>350 \times 10^{9} / \mathrm{L}\right)$ and neutrophil count $\left(>7,500 \times 10^{9} / \mathrm{L}\right)$ had predictive value with respect to $\mathrm{pCR}$. Sun et al. [10] demonstrated that both elevated NLR (>3.05) and PLR (145.98) were independent predictors of a good response to neoadjuvant CRT. Andras et al. [23] reported that NLR is a simple and cost-effective marker for predicting neoadjuvant treatment response in LARC, with a cut-off value of 4.5. In contrast, Dudani et al. [25] analyzed 1,237 rectal cancer patients who underwent neoadjuvant CRT, and showed that neither pretreatment PLR nor NLR predicted pCR. However, some studies have focused on changes in serologic markers before and after neoadjuvant CRT. Lee et al. [26] reported that changes in PLR after neoadjuvant CRT could be used as a predictive marker for pCR in rectal cancer patients. In their analysis, the degree of increase in PLR after CRT was the strongest predictor of pCR among various serologic markers. Lai et al. [27] demonstrated that pretreatment PLR and NLR did not correlate with pCR, but a $21.5 \%$ change in NLR after neoadjuvant CRT was a predictor of poor $\mathrm{PCR}$.

In terms of survival rate, many studies have investigated whether pretreatment serologic markers, such as PLR and NLR, are associated with long-term oncologic survival as well as pCR in rectal cancer patients who underwent neoadjuvant CRT. A retrospective study by Ke et al. [9] revealed that a pre-CRT elevated PLR (>188) was an independent prognostic factor for poor DFS, but not for OS, and that a pre-CRT elevated NLR (>3.5) was an independent prognostic factor for both poor DFS and OS. Zhang et al. [28] reported that a high NLR $(\geq 2.3)$ was associated with unfavorable OS and DFS, and they also reported that NLR was the most effective prognostic marker according to time-dependent ROC analysis. In a study where the cut-off value of NLR was the same as ours, elevated NLR was associated with poor OS, but not with DFS. In addition, NLR did not reflect the response to neoadjuvant CRT [29]. In contrast, there have been several studies showing that pretreatment serologic markers are not related to the survival rate of rectal cancer patients who underwent neoadjuvant CRT followed by curative resection. Dudani et al. [25] conducted a study by setting the cut-off values of PLR and NLR to 150 and 4, respectively, and reported that PLR and NLR were not independent prognostic factors of DFS or OS. Portale et al. [11] found neither PLR nor NLR to be associated with survival in rectal cancer patients. In our analysis, both PLR and NLR showed a correlation with OS, but not with DFS. Further analysis also showed that the cases with a high NLR and PLR showed a poor tendency for DFS compared to those with a low NLR and PLR, but this was not statistically significant. In addition, these serologic markers did not show any association with local recurrence or distant metastasis. In multivariate analysis, only PLR, among the 2 markers, was found to be an independent prognostic factor for OS. Because the cut-off values of these laboratory markers differ from study to study, caution should be taken when generalizing the findings of 
individual studies. According to a recent meta-analysis by Hamid et al. [30] which included rectal cancer patients, elevated NLR was an independent factor for poor OS (HR, 2.07; 95\% CI, 1.57-2.74; $\mathrm{P}<0.001)$ and DFS (HR, 1.80; 95\% CI, 1.42-2.28, $\mathrm{P}<0.001)$, and the results were the same in the subgroup analysis of patients who received neoadjuvant CRT. In addition, the study demonstrated that the prognostic effect of NLR was superior to that of PLR when comparing the relative HR and other markers.

Our study has several limitations. First, the present study may have selection bias due to its retrospective design. Second, the total number of patients included in this study was relatively small. Third, because the data were obtained from a single center, it may not include the characteristics of various types of patients. Fourth, information on gene profiling and some important pathological reports, such as lymphovascular invasion and perineural invasion, were not assessed because old medical records did not include such additional pathologic features. Fifth, patient factors, such as comorbidities and nutritional status, were not analyzed. Therefore, additional well-designed large-scale studies are needed to confirm the prognostic effect of inflammatory markers with a high level of evidence.

In conclusion, pretreatment PLR may be considered as a potential biomarker for predicting OS in patients with locally advanced rectal cancer who received neoadjuvant CRT followed by curative resection. Although the TNM staging system is still the strongest prognostic factor for rectal cancer, pretreatment laboratory inflammatory markers and tumor markers may provide helpful additional prognostic information. If future related large-scale studies will prove the utility of these economically advantageous serologic biomarkers, it may help clinicians in treatment decisionmaking for locally advanced rectal cancer patients.

\section{CONFLICT OF INTEREST}

No potential conflict of interest relevant to this article was reported.

\section{FUNDING}

None.

\section{REFERENCES}

1. Bray F, Ferlay J, Soerjomataram I, Siegel RL, Torre LA, Jemal A. Global cancer statistics 2018: GLOBOCAN estimates of incidence and mortality worldwide for 36 cancers in 185 countries. CA Cancer J Clin 2018;68:394-424.

2. Benson AB, Venook AP, Al-Hawary MM, Arain MA, Chen YJ, Ciombor KK, et al. NCCN guidelines insights: rectal cancer, version 6.2020. J Natl Compr Canc Netw 2020;18:806-15.

3. Siegel RL, Miller KD, Jemal A. Cancer statistics, 2020. CA Cancer J Clin 2020;70:7-30.
4. Dolan RD, McSorley ST, Park JH, Watt DG, Roxburgh CS, Horgan PG, et al. The prognostic value of systemic inflammation in patients undergoing surgery for colon cancer: comparison of composite ratios and cumulative scores. Br J Cancer 2018;119:40-51.

5. Watt DG, Ramanathan ML, McSorley ST, Walley K, Park JH, Horgan PG, et al. Clinicopathological determinants of an elevated systemic inflammatory response following elective potentially curative resection for colorectal cancer. Ann Surg Oncol 2017;24: 2588-94.

6. Dolan RD, Laird BJ, Horgan PG, McMillan DC. The prognostic value of the systemic inflammatory response in randomised clinical trials in cancer: a systematic review. Crit Rev Oncol Hematol 2018;132:130-7.

7. Ozawa T, Ishihara S, Nishikawa T, Tanaka T, Tanaka J, Kiyomatsu $\mathrm{T}$, et al. The preoperative platelet to lymphocyte ratio is a prognostic marker in patients with stage II colorectal cancer. Int J Colorectal Dis 2015;30:1165-71.

8. Huang XZ, Chen WJ, Zhang X, Wu CC, Zhang CY, Sun SS, et al. An elevated platelet-to-lymphocyte ratio predicts poor prognosis and clinicopathological characteristics in patients with colorectal cancer: a meta-analysis. Dis Markers 2017;2017:1053125.

9. Ke TM, Lin LC, Huang CC, Chien YW, Ting WC, Yang CC. High neutrophil-to-lymphocyte ratio and platelet-to-lymphocyte ratio predict poor survival in rectal cancer patients receiving neoadjuvant concurrent chemoradiotherapy. Medicine (Baltimore) 2020; 99:e19877.

10. Sun Y, Huang Z, Chi P. An inflammation index-based prediction of treatment response to neoadjuvant chemoradiotherapy for rectal mucinous adenocarcinoma. Int J Clin Oncol 2020;25:1299307.

11. Portale G, Cavallin F, Valdegamberi A, Frigo F, Fiscon V. Plateletto-lymphocyte ratio and neutrophil-to-lymphocyte ratio are not prognostic biomarkers in rectal cancer patients with curative resection. J Gastrointest Surg 2018;22:1611-8.

12. Ruggeri ZM, Mendolicchio GL. Adhesion mechanisms in platelet function. Circ Res 2007;100:1673-85.

13. Jenne CN, Kubes P. Platelets in inflammation and infection. Platelets 2015;26:286-92.

14. Haemmerle M, Stone RL, Menter DG, Afshar-Kharghan V, Sood AK. The platelet lifeline to cancer: challenges and opportunities. Cancer Cell 2018;33:965-83.

15. Gu D, Szallasi A. Thrombocytosis portends adverse prognosis in colorectal cancer: a meta-analysis of 5,619 patients in 16 individual studies. Anticancer Res 2017;37:4717-26.

16. Belluco C, Forlin M, Delrio P, Rega D, Degiuli M, Sofia S, et al. Elevated platelet count is a negative predictive and prognostic marker in locally advanced rectal cancer undergoing neoadjuvant chemoradiation: a retrospective multi-institutional study on 965 patients. BMC Cancer 2018;18:1094.

17. Ohtani H. Focus on TILs: prognostic significance of tumor infiltrating lymphocytes in human colorectal cancer. Cancer Immun 2007;7:4. 
18. Ocana A, Nieto-Jiménez C, Pandiella A, Templeton AJ. Neutrophils in cancer: prognostic role and therapeutic strategies. Mol Cancer 2017;16:137.

19. Sell NM, Qwaider YZ, Goldstone RN, Cauley CE, Cusack JC, Ricciardi R, et al. Ten-year survival after pathologic complete response in rectal adenocarcinoma. J Surg Oncol 2021;123:293-8.

20. Habr-Gama A, Perez RO, Nadalin W, Sabbaga J, Ribeiro U Jr, Silva e Sousa AH Jr, et al. Operative versus nonoperative treatment for stage 0 distal rectal cancer following chemoradiation therapy: long-term results. Ann Surg 2004;240:711-7.

21. Dossa F, Chesney TR, Acuna SA, Baxter NN. A watch-and-wait approach for locally advanced rectal cancer after a clinical complete response following neoadjuvant chemoradiation: a systematic review and meta-analysis. Lancet Gastroenterol Hepatol 2017; 2:501-13.

22. Park IJ, Lee JL, Yoon YS, Kim CW, Lim SB, Yu CS, et al. Oncologic outcomes of organ preserving approaches in patients with rectal cancer treated with preoperative chemoradiotherapy. Ann Coloproctol 2019;35:65-71.

23. Andras D, Crisan D, Craciun R, Nemes A, Caziuc A, Drasovean R, et al. Neutrophil-to-lymphocyte ratio: a hidden gem in predicting neoadjuvant treatment response in locally advanced rectal cancer? J BUON 2020;25:1436-42.

24. Policicchio A, Mercier J, Digklia A, Voutsadakis IA. Platelet and neutrophil counts as predictive markers of neoadjuvant therapy efficacy in rectal cancer. J Gastrointest Cancer 2019;50:894-900.
25. Dudani S, Marginean H, Tang PA, Monzon JG, Raissouni S, Asmis TR, et al. Neutrophil-to-lymphocyte and platelet-to-lymphocyte ratios as predictive and prognostic markers in patients with locally advanced rectal cancer treated with neoadjuvant chemoradiation. BMC Cancer 2019;19:664.

26. Lee JH, Song C, Kang SB, Lee HS, Lee KW, Kim JS. Predicting pathological complete regression with haematological markers during neoadjuvant chemoradiotherapy for locally advanced rectal cancer. Anticancer Res 2018;38:6905-10.

27. Lai S, Huang L, Luo S, Liu Z, Dong J, Wang L, et al. Systemic inflammatory indices predict tumor response to neoadjuvant chemoradiotherapy for locally advanced rectal cancer. Oncol Lett 2020;20:276370 .

28. Zhang Y, Liu X, Xu M, Chen K, Li S, Guan G. Prognostic value of pretreatment systemic inflammatory markers in patients with locally advanced rectal cancer following neoadjuvant chemoradiotherapy. Sci Rep 2020;10:8017.

29. Shen L, Zhang H, Liang L, Li G, Fan M, Wu Y, et al. Baseline neutrophil-lymphocyte ratio $(\geq 2.8)$ as a prognostic factor for patients with locally advanced rectal cancer undergoing neoadjuvant chemoradiation. Radiat Oncol 2014;9:295.

30. Hamid H, Davis GN, Trejo-Avila M, Igwe PO, Garcia-Marín A. Prognostic and predictive value of neutrophil-to-lymphocyte ratio after curative rectal cancer resection: a systematic review and meta-analysis. Surg Oncol 2021;37:101556. 